\title{
Clinical outcome of dysglycaemia in children admitted to pediatric ward at a tertiary care hospital - a prospective study
}

\author{
Polasa $\mathbf{R}^{1}$, Gudi $\mathbf{P}^{2}$ \\ ${ }^{1}$ Dr Ramesh Polasa, Associate Professor, ${ }^{2}$ Dr. Pratap Gudi, Associate Professor; both authors are affiliated with Department \\ of Pediatrics, Kakatiya Medical College, Warangal, Telangana, India.
}

Address for Correspondence: Dr. Ramesh Polasa, Associate Professor, Department of Pediatrics, Kakatiya Medical College, Warangal, Telangana, India.

\begin{abstract}
Background: Dysglycaemia (hyper- or hypoglycaemia) in critically ill children has been associated with poor outcome. Objective: comparison of clinical outcomes in children admitted to Emergency unit of MGM Hospital, Warrangal for acute medical conditions and presenting with euglycaemia or dysglycaemia. Methods: Five hundred and twenty five subjects aged between 4 months and 12 years were screened out of whom 330 (205 with euglycaemia and 125 with dysglycaemia) were enrolled. Results: In the dysglycaemia group, 28 (13\%) subjects had hypoglycemia and 97 (87\%) had hyperglycemia. Overall, there were 101 complications in 125 subjects. The number of subjects with complications was significantly higher in dysglycaemia group (46\%) compared to euglycaemia group (8\%). Fifty subjects died out of whom 30 had dysglycaemia. Subjects with dysglycaemia were 3 times (95\% CI: 1.5-6.0) more likely to die and 4.8 times (95\% CI: 3.1-7.5) more likely to develop complications. Conclusion: Dysglycaemia is associated with increased morbidity and mortality in children with acute medical conditions.
\end{abstract}

Keywords: Dysglycaemia, Pediatric age group, Euglycaemia, Hyperglycaemia

\section{Introduction}

In children, the response to the stress of an acute illness can present as abnormal blood glucose [1, 2]. It was found that $3.8 \%$ of children and adolescents presenting to the emergency room in Norfolk (VA) had hyperglycaemia [2]. Hyperglycaemia was not associated with a specific diagnosis category but reflected a greater severity of the underlying condition. In contrast, Elusiyan et al. observed that $6.4 \%$ of pediatric subjects admitted to a hospital in Nigeria had hypoglycaemia [3]. Similarly, hypoglycaemia was observed across a large range of conditions and was associated with increased mortality. Overall, hypoglycaemia as well as hyperglycaemia and, in addition, increased glucose variability [4-7] have been found to be associated with increased morbidity and mortality rates in acutely ill children in a variety of settings.

Manuscript received: $10^{\text {th }}$ October 2016

Reviewed: $16^{\text {th }}$ October 2016

Author Corrected: $24^{\text {th }}$ October 2016

Accepted for Publication: $30^{\text {th }}$ October 2016
There is presently no data on abnormal blood glucose concentrations as well as on their potential relationship with patient outcome in children with acute illnesses admitted to the Pediatric Emergency Unit (PEU) in our teaching hospital. Using a prospective study, the objectives of this study was to assess dysglycaemia as a prognostic factor for adverse clinical outcome in children admitted to MGM Hospital, Warangal.

\section{Methods}

The study was conducted on consecutive subjects presenting over a six -month period between December 2014- June 2015. All children admitted at Pediatric Emergency Unit (PEU) of MGM Hospital, Warrangal with acute illness participated in this study. Inclusion criteria were admission to PEU with acute illness and age between 3 months and 12 years. Exclusion criteria were known history of diabetes mellitus; infusion of dextrose containing fluids up to 2 hours prior to admission; and 
intake of steroids within 72 hours of admission. Data were collected daily between 8 a.m. and 8 p.m. All subjects admitted to PEU with acute illnesses had their blood glucose checked with glucometer (Lifescan Company, Milpitas, CA, USA). The range of the glucose concentrations measured with glucometer is $0.6-$ $33.3 \mathrm{mmol} / \mathrm{L}(10-600 \mathrm{mg} / \mathrm{dL})$.

A difference of less than $10 \%$ between that measure by glucometer and that by the biochemistry laboratory was regarded as acceptable. Hyperglycaemia and hypoglycaemia were defined as blood glucose greater than $8.3 \mathrm{mmol} / \mathrm{L}(150 \mathrm{mg} / \mathrm{dL})$ and less than $2.5 \mathrm{mmol} / \mathrm{L}$ (45 mg/dL), respectively [4].

A subject with acute illness and abnormal blood glucose (dysglycaemia group) was matched to the next patient with normal blood glucose (euglycaemia group) but with the same diagnosis category and age group (age from 3 months to $<2$ years, 2 to $<5$ years, and 5 to 12 years) in order to better compare the characteristics of the conditions associated with dysglycemia.
They were followed up till discharge or death. On discharge, their folders were collected and case record form was completed for final diagnosis and complications developed including death. Subjects in whom hyperglycemia did not resolve or worsened after initiating specific treatment of the primary condition were treated with regular insulin and monitored closely [9]. In contrast, those with hypoglycaemia were managed immediately according to our local protocol.

Our hospital protocol calls for an initial bolus intravenous (iv), intraosseous (io), or rectal dextrose or intramuscular (im) glucagon followed by i.v. $10 \%$ dextrose at maintenance. Feeding is resumed as soon as possible. Necessary supportive management was given to each patient.

Risks of adverse outcomes in both groups were calculated and compared using risk ratios with 95\% confidence intervals. Student's -test was used to assess differences in the means of continuous variables and significant levels were assessed using value of $<0.05$.

\section{Results}

Over the course of 6 months, 125 subjects with dysglycaemia were recruited and matched for age group and diagnosis category with 205 euglycaemic subjects. In total, 525 subjects were screened ( 205 euglycemic, 28 hypoglycaemic, and 125 hyperglycaemic).

The median age of the subjects was 24 months (range: 4-144 months). The proportion of males and females was similar ( $65 \%$ boys in the euglycemic group and $50 \%$ boys in the dysglycaemia group). In the dysglycaemia group, 28 (22\%) out of 125 subjects had hypoglycemia and 97 (88\%) had hyperglycemia (table 1).

In keeping with the case-matching design of the study, the age distribution and the diagnosis categories were identical in euglycaemic and dysglycaemic subjects. Table 2 describes the medical conditions observed in the subjects enrolled in the study. In keeping with the case-matching design of the study, the age distribution and the diagnosis categories were identical in euglycaemic and dysglycaemic subjects.

Table-1: Demographic characteristics of cases.

\begin{tabular}{|c|c|c|c|}
\hline \multirow{2}{*}{ Age (months) } & Euglycaemia (\%) & \multicolumn{2}{|c|}{ Dysglycaemia (\%) } \\
\cline { 3 - 4 } & & Hypoglycemia & Hyperglycemia \\
\hline $4.0-23.9$ & $101(47)$ & $17(61)$ & $34(35)$ \\
\hline $24.0-59.9$ & $54(27)$ & $5(18)$ & $33(34)$ \\
\hline $60.0-144.0$ & $50(26)$ & $6(21)$ & $29(30)$ \\
\hline Total & $\mathbf{2 0 5}$ & $\mathbf{2 8}$ & $\mathbf{9 7}$ \\
\hline
\end{tabular}


Data are given as (\% of the patients with euglycemia or with dysglycemia (all, hypoglycemia and hyperglycemia).

Overall, there were complications observed in the subjects, which includes disseminated intravascular coagulation, acute respiratory failure, intraventricular hemorrhage, cortical blindness, hemiparesis, haemoglobinuria, heart failure, hypoglycaemia, intestinal perforation, and repeated convulsions. The number of subjects with complications was significantly higher in the dysglycaemia group (46\%) compared to the euglycaemia group (8\%). In the dysglycemia group, the proportion of subjects with complications was similar for hypoglycemia and hyperglycemia.

Table-2: Categories of diagnosis in children presenting with acute medical conditions

\begin{tabular}{|c|c|c|}
\hline Diagnosis & Frequency & Percent (\%) \\
\hline Severe malaria & 163 & 6 \\
\hline Gastroenteritis & 23 & 10 \\
\hline Septicaemia & 41 & 4 \\
\hline Typhoid fever & 15 & 13 \\
\hline Meningitis & 17 & 13 \\
\hline Diarrhea and vomiting & 46 & 7 \\
\hline Acute respiratory disease & 46 & 2 \\
\hline
\end{tabular}

Others: urinary tract infection, tonsillitis, and otitis media.

The majority of the subjects $(88 \%)$ had no complications at discharge and $8(2 \%)$ were discharged with one or more complications. Six of them were in the dysglycemia group (hyperglycemia). Those who were discharged with complications were followed up till resolution. At the end of the study, 50 subjects had died.

Death was statistically more common in subjects with dysglycaemia at admission than in euglycaemic subjects. Among the 40 subjects with dysglycemia who died, 30 presented with hyperglycemia ( $=11 \%$ of the subjects with hyperglycemia) and 10 with hypoglycemia ( $=36 \%$ of the subjects with hypoglycemia). The risk of dying or of developing complications for subjects with dysglycaemia was, respectively, 3 times (95\% CI: 1.5-6.0) and 4.8 times higher (95\% CI: 3.1-7.5) than for euglycaemic subjects.

\section{Discussion}

The results of our study show that both hypoglycemia and hyperglycemia reflect increased severity of an acute medical condition in children presenting to the Emergency ward.

These figures are similar to findings in other studies. Elusiyan et al [3] found out that presence of hypoglycaemia at admission was associated with death and dying within 24 hours of admission. Moreover, Osier et al. found out that mortality for children with abnormal blood glucose was $34.2 \%$ compared to $7.6 \%$ in euglycaemic children admitted to an emergency ward [4].
Furthermore, Dungan et al. demonstrated that mortality in hyperglycaemic children was about twice as much as that in euglycaemic children even though the correlation between blood sugar and mortality could not be established [8].

Additionally, a study in Mozambique revealed that mortality in children with acute medical disease was $16.3 \%$ in children with hypoglycaemia compared to $3.2 \%$ in those who were normoglycaemic [9,10]. Accordingly, we found out that subjects with dysglycaemia were 3 times more likely to die and 4.8 times more likely to have complications than those with 
euglycemia. Solomon et al. [10] found a relative risk of 5.8 for mortality in children with acute medical conditions and hypoglycaemia in Mozambique. The relative risk of dying of hypoglycaemia in the Mozambique study was about twice that of this study. Overall, the value of dysglycemia as a prognostic factor for mortality and morbidity seems well established.

In our study, the commonest disease found to be associated with dysglycaemia was severe malaria. The other common disease conditions we encountered were diarrhea and vomiting, acute respiratory diseases, and septicaemia. Various diseases have been established in other studies to be associated with abnormal blood glucose including severe malaria, gastroenteritis, septicaemia, pneumonia, and acute febrile seizures $\quad$ [1117].

Looking more in depth at malaria, the most common condition identified in this study, the link between dysglycaemia and severity of the disease is not completely understood. In a mostly pediatric population, Eltahir et al. [18] observed hyperglycemia in severe cerebral malaria which was secondary to an increase in insulin resistance and possibly relted to a decrease in insulin production. On the other hand, hypoglycemia is also commonly reported in children with severe malaria. Abdallah et al. [21] observed hypoglycemia in $12 \%$ of adult subjects receiving intravenous quinine for severe malaria. However, Kawo et al. [22] did not observe significant changes in blood glucose in subjects with falciparum malaria receiving intravenous administration of quinine.

Putting together, these data suggest that early recognition of dysglycaemia in subjects with acute illness should prompt the health professional to adopt a more aggressive management approach.

Several issues remain to be clarified. First, there is a need for a better definition of the severity of hypoglycemia with regard to the increased risk of morbidity and mortality [23]. Second, whether correction of dysglycemia in addition to aggressive management of the underlying condition is beneficial to the subject remains unclear. However, administration of glucose is recommended by the WHO and advocated by some authors $[19,20]$. A recent review on the effect of acute hypoglycemia management in children suggests that the evidence remains insufficient and that long term studies are needed [24, 25].

A strength of our study is the prospective, case matched cohort design. Previous studies that compared the health outcomes according to glycaemia at admission did include all patients without attempt to matching. As dysglycaemia is much less common than euglycaemia, this resulted in groups of very different sizes and etiologies. While the latter design allows for a description of the epidemiology of subjects with dysglycemia, our design is stronger when it comes to comparing the outcomes.

\section{Conclusion}

Abnormal blood glucose (dysglycaemia) is associated with increased morbidity and mortality in children with acute medical conditions on admission. Fatal outcome was more common in those who are hypoglycemic at admission.

Acknowledgments: We wish to thank all the staff members of department of Pediatrics, Kakatiya Medical College, warangal for their kind co-operation during the study.

Funding: Nil, Conflict of interest: None initiated, Perission from IRB: Yes

Ethical clearance: Obtained from Institutional ethics committee

\section{References}

1. Bhisitkul DM, Vinik AI, Morrow AL, She JX, Shults J, Powers AC, Maclaren NK. Prediabetic markers in children with stress hyperglycemia. Archives of Pediatrics and Adolescent Medicine 1996; 150 (9): 936941.

2. Bhisitkul DM, Morrow AL, Vinik AI, Shults J, Layland JC, Rohn R. Prevalence of stress hyperglycemia among patients attending a pediatric emergency department. Journal of Pediatrics 1994; 124 (4): 547-551.

3. Elusiyan JB, Adejuyigbe EA, Adeodu OO. Hypoglycaemia in a Nigerian paediatric emergency 
ward. J Trop Pediatr. 2006 Apr;52(2):96-102. Epub 2005 Sep 16.

4. Osier FH, Berkley JA, Ross A, Sanderson F, Mohammed S, Newton CR. Abnormal blood glucose concentrations on admission to a rural Kenyan district hospital: prevalence and outcome. Arch Dis Child. 2003 Jul; 88 (7):621-5.

5. Kwiatkowski D, Hill AV, Sambou I, Twumasi P, Castracane J, Manogue KR, Cerami A, Brewster DR, Greenwood BM.TNF concentration in fatal cerebral, non-fatal cerebral, and uncomplicated Plasmodium falciparum malaria Lancet. 1990 Nov 17; 336 (8725):1201-4.

6. Wintergerst KA, Buckingham B, Gandrud L, Wong BJ, Kache S, Wilson DM.Association of hypoglycemia, hyperglycemia, and glucose variability with morbidity and death in the pediatric intensive care unit. Pediatrics. $2006 \mathrm{Jul} ; 118(1): 173-9$.

7. Langdon DR, Stanley CA, Sperling MA. Hypoglycemia in the infant and child. In: Sperling MA, editor. Pediatric endocrinology. 3rd edition. Philadelphia: Saunders Elsevier; 2008. pp. 422-423.

8. Dungan KM, Braithwaite SS, Preiser JC. Stress hyperglycaemia. Lancet. 2009 May 23;373(9677):1798807. doi: 10.1016/S0140-6736(09)60553-5.

9. Gupta P, Natarajan G, Agarwal KN. Transient hyperglycemia in acute childhood illnesses: to attend or ignore? Indian J Pediatr. 1997 Mar-Apr;64(2):205-10.

10. Solomon T, Felix JM, Samuel M, Dengo GA, Saldanha RA, Schapira A, Phillips RE. Hypoglycaemia in paediatric admissions in Mozambique. Lancet. 1994 Jan 15;343(8890):149-50.

11. English M, Sauerwein R, Waruiru C, Mosobo M, Obiero J, Lowe B, Marsh K. Acidosis in severe childhood malaria. QJM. 1997 Apr;90(4):263-70.

12. Thien HV, Kager PA, Sauerwein HP. Hypo-glycemia in falciparum malaria: is fasting an unrecognized and insufficiently emphasized risk factor? Trends Parasitol.2006Sep;22(9):410-5.Epub2006Jul 12.

13. Zijlmans W, van Kempen A, Ackermans M, de Metz

J, Kager P, Sauerwein H. Glucose kinetics during fasting in young children with severe and non-severe malaria in suriname. Am J Trop Med Hyg. 2008;79 (4): 605-12.

14. Waller D, Krishna S, Crawley J, Miller K, Nosten F, Chapman D, ter Kuile FO, Craddock C, Berry C, Holloway PA, et al. Clinical features and outcome of severe malaria in Gambian children. Clin Infect Dis. 1995 Sep;21(3):577-87.

15. Bjerre JV. [Stress hyperglycemia in a child with severe acute gastroenteritis]. Ugeskr Laeger. 2002 Nov 18;164(47):5524-5.

16. de Groof F, Joosten KF, Janssen JA, de Kleijn ED, Hazelzet JA, Hop WC, Uitterlinden P, van Doorn J, Hokken-Koelega AC. Acute stress response in children with meningococcal sepsis: important differences in the growth hormone/insulin-like growth factor I axis between nonsurvivors and survivors. J Clin Endocrinol Metab. 2002;87(7):3118-24.

17. van Waardenburg DA, Jansen TC, Vos GD, Buurman WA. Hyperglycemia in children with meningococcal sepsis and septic shock: the relation between plasma levels of insulin and inflammatory mediators. J Clin Endocrinol Metab. 2006;91(10): 391621.

18. Don M, Valerio G, Korppi M, Canciani M. Hyperand hypoglycemia in children with community-acquired pneumonia. J Pediatr Endocrinol Metab. 2008 Jul;21 (7): 657-64.

19. Eltahir EM, El Ghazali G, A-Elgadir TM, A-Elbasit IE, Elbashir MI, Giha HA. Raised plasma insulin level and homeostasis model assessment (HOMA) score in cerebral malaria: evidence for insulin resistance and marker of virulence. Acta Biochim Pol. 2010;57 (4):51320. Epub 2010 Dec 6.

20. Manning L, Laman M, Davis WA, Davis TM. Clinical features and outcome in children with severe Plasmodium falciparum malaria: a meta-analysis. PLoS One. 2014 Feb 6; 9 (2):e86737. doi: 10.1371/journal. pone. 0086737. eCollection 2014.

21. Abdallah TM, Elmardi KA, Elhassan AH, Omer MB, Elhag MS, Desogi MA, Siddig MF, Adam I. Comparison of artesunate and quinine in the treatment of severe Plasmodium falciparum malaria at Kassala hospital, 
Sudan. J Infect Dev Ctries. 2014 May 14; 8 (5): 611-5. doi: 10.3855/jidc.3813.

22. Kawo NG, Msengi AE, Swai AB, Orskov H, Alberti $\mathrm{KG}, \mathrm{McLarty}$ DG. The metabolic effects of quinine in children with severe and complicated Plasmodium falciparum malaria in Dar es Salaam. Trans R Soc Trop Med Hyg. 1991;85(6):711-3.

23. Willcox ML, Forster M, Dicko MI, Graz B, MayonWhite R, Barennes H. Blood glucose and prognosis in children with presumed severe malaria: is there a threshold for 'hypoglycaemia'? Trop Med Int Health.
2010 Feb;15(2):232-40. doi: 10.1111/j.1365-3156.2009. 02444.x. Epub 2009 Dec 4.

24. Sambany E, Pussard E, Rajaonarivo C, Raobijaona H, Barennes H. Childhood dysglycemia: prevalence and outcome in a referral hospital. PLoS One. 2013 May 31; 8 (5):e65193. doi: 10.1371/journal.pone.0065193. Print 2013.

25. Achoki R, Opiyo N, English M. Mini-review: Management of hypoglycaemia in children aged 0-59 months. J Trop Pediatr. 2010 Aug;56(4):227-34. doi: 10.1093/tropej/fmp109. Epub 2009 Nov 23.

\section{How to cite this article?}

Polasa R, Gudi P. Clinical outcome of dysglycaemia in children admitted to pediatric ward at a tertiary care hospital- a prospective study. J PediatrRes.2017;4(01):29-34.doi:10.17511/ijpr.2017.i01.06 